\title{
Padé discretization for linear systems with polyhedral Lyapunov functions
}

\author{
Francesco Rossi, Patrizio Colaneri, Robert Shorten.
}

\begin{abstract}
This paper has been motivated by the need to assess the preservation of polyhedral Lyapunov functions for stable continuous-time linear systems under numerical discretization of the transition matrix. This problem arises when discretizing linear systems in such a manner as to preserve a certain type of stability of the discrete time approximation. Our main contribution is to show that a continuous-time system (with distinct eigenvalues) and its Padé discretization (of any order and sampling) always share at least one common piecewise linear (polyhedral) Lyapunov function.
\end{abstract}

Index Terms-Stability of linear systems, discretization, Lyapunov function.

\section{INTRODUCTION}

$\mathbf{T}$ HE investigation of the properties of linear systems when passing from the continuous-time analysis to the discrete-time one has been subject of particular attention in the literature of control theory. For linear time-invariant (LTI) systems, this procedure is almost completely understood, and forms a fundamental basis, both for the design of control systems, and for numerical simulation.

Recently, in the context of the study of switched linear systems, several papers have considered the problem of discrete approximations to continuous-time switched linear systems [1], [2], [3], [4]. The theory of switched linear systems is a relatively new field of research where the knowledge of the shared properties between continuous-time and discrete-time systems is completely absent. This constitutes a significant gap in the literature as engineering practice requires discrete time implementations of engineering designs. Consequently it is possible to formulate many basic questions that, remarkably, have yet to be addressed in any form.

One such question concerns preservation of stability. For LTI systems, many discrete approximations are known to preserve quadratic stability. Since quadratic and exponential stability are indistinguishable concepts for LTI systems, the story, in this case, is complete. A fundamental issue for the discretisation of switched systems is that quadratic stability is only a sufficient condition for exponential stability. A more complete characterisation of exponential stability requires the study of piecewise-linear and piecewise quadratic Lyapunov functions [5]. Thus, for switched systems, the notion of stability preservation goes beyond the notion of quadratic Lyapunov functions, and requires the study of more elaborate Lyapunov

F. Rossi is with Lab LSIS, Université Paul Cézanne, Marseille, France, E-mail: francesco.rossi@1sis.org.

P. Colaneri is with Politecnico di Milano, Dipartimento di Elettronica e Informazione, Milan, Italy, E-mail: colaneri@elet.polimi.it.

R. Shorten are with National University of Ireland, Hamilton Institute, Maynooth, Co. Kildare, Ireland, E-mail: robert.shorten@nuim.ie. functions. Our paper is motivated by this observation, and takes a first small step in this direction. Among discretization methods we will consider the Padé transformation, which is widely used in real applications and intensively studied from the numerical viewpoint: [6], [7]. Such a study is well motivated, as diagonal Padé approximations are a method of choice amongst control engineers. (the Tustin or Bilinear transform is an example of a diagonal Padé approximation). Our primary focus in this paper are polyhedral Lyapunov functions. Such functions are known to be nonconservative in the analysis of stability under arbitrary switching for polytopic and switched systems We shall ask for what class of discretisations are such norms preserved, and in this context we shall show that a stable continuous-time system and its Padé discretized version of order $p \geq 1$ (for any sampling time) always share such a function. This does not contradict previous results in [8], where it is proven, through a counterexample, that a given polyhedral Lyapunov function in continuous-time may be not a Lyapunov function for the sampled discrete-time system obtained via the particular class of bilinear transformations with fixed sampling time.

The question as to what properties are preserved under such discretisations appears to be a new problem and, despite its importance, has not yet received the attention it deserves. Roughly speaking, our results show that certain types of polyhedral Lyapunov functions are preserved under Padé discretisations of LTI systems. The importance of this results follows from the fact that, for switched linear systems, piecewise linear Lyapunov functions are known to be non-conservative for establishing the studying the stability of switched linear systems. In this context, we believe that our main result is the starting point to get a deeper understanding on the effect of discretization of switched systems, also in the study of preservation of optimality criteria [9].

\section{PREliminaries}

Consider a linear autonomous system

$$
\dot{x}(t)=A_{c} x(t)
$$

where $x(t) \in \mathcal{R}^{n}$ and assume that the system is asymptotically stable, i.e. matrix $A_{c}$ is Hurwitz (all eigenvalues in the open left half of the complex plane). It is well known that the motion of the state, associated with an initial state $x(0)=x_{0}$, can be written as $x(t)=e^{A_{c} t} x_{0}$. The exponential matrix $e^{A_{c} t}$ can be numerically approximated in a variety of different ways. In this paper we focus on the most popular one, that is diagonal Padé approximation approximation of $p^{\text {th }}$ order, see e.g. [6], [7]. This operator is well known to engineers and is commonly 
used by both the control and signal processing community. To be precise, taking a sampling time $h$, the $p^{t h}$ order Padé discretization of $e^{A_{c} h}$ is defined as

$$
\begin{aligned}
& A_{d}(h)=Z\left(A_{c} h\right) Z\left(-A_{c} h\right)^{-1} \\
& Z(X)=\sum_{i=0}^{p} c_{i} X^{i}, \quad c_{i}=\frac{(2 p-i) ! p !}{(2 p) ! i !(p-i) !}
\end{aligned}
$$

Hence, it is possible to associate with system (1), its discrete approximation

$$
x_{d}(k+1)=A_{d}(h) x_{d}(k)
$$

where $x_{d}(k)$ approximates $x(k h)=\left(e^{A_{c} h k}\right) x_{0}$. It is well known that the Padé discretization preserves the stability properties. As a matter of fact, $A_{c}$ is Hurwitz if and only if $A_{d}$ is Schur stable (all eigenvalues inside the open unit disc), for any given sampling times $h>0$. Moreover, the eigenvalues of $A_{c}$ and $A_{d}(h)$ are related by the same transformation induced by (2), and the eigenstrucure is preserved. If $\lambda$ is an eigenvalue of $A_{c}$ associated with an eigenvector $\bar{x}$, then $z=Z(\lambda h) Z(-\lambda h)^{-1}$ is an eigenvalue of $A_{d}(h)$ associated with the same eigenvector $\bar{x}$. Even more, the transformation is basis independent, i.e.

$$
\begin{aligned}
& Z\left(T A_{c} T^{-1} h\right) Z\left(-T A_{c} T^{-1} h\right)^{-1}= \\
& =T Z\left(A_{c} h\right) Z\left(-A_{c} h\right)^{-1} T^{-1}=T A_{d}(h) T^{-1}
\end{aligned}
$$

Finally, if $T A_{c} T^{-1}$ is a Jordan form with distinct eigenvalues ${ }^{1}$ for $A_{c}$, then $T A_{d}(h) T^{-1}$ is a Jordan form for $A_{d}(h)$. A particular Padé transformation is the celebrated bilinear transformation (or Tustin transformation), that is given by (2) with $p=1$, i.e.

$$
A_{d}(h)=\left(I+\frac{h}{2} A_{c}\right)\left(I-\frac{h}{2} A_{c}\right)^{-1}
$$

\section{QUADRATIC AND POLYHEDRAL LYAPUNOV FUNCTIONS}

Consider system (1) and its Padé discretization (3). Assume that $A_{c}$ is Hurwitz stable, so that $A_{d}(h)$ is Schur stable for each $h>0$. We consider the 2-measure of a square matrix $X$ as $\mu_{2}(X)=\frac{1}{2} \lambda_{\max }\left(X+X^{\prime}\right)$ and the 2-norm as $\|X\|_{2}=$ $\sqrt{\lambda_{\max }\left(X^{\prime} X\right)}$. Also, letting $X_{i j}$ be the entries of $X$, we define the $\infty$-measure as $\mu_{\infty}(X)=\max _{i}\left(X_{i i}+\sum_{j \neq i}\left|X_{i j}\right|\right)$ and the $\infty$-norm as $\|X\|_{\infty}=\max _{\mathbf{i}} \sum_{j}\left|X_{i j}\right|$. The main results relating stability are recalled below, see e.g. [10].

\section{Lemma 1:}

(i) $A_{c}$ is Hurwitz stable if and only if there exists a full column rank matrix $W_{c} \in \mathcal{R}^{N \times n}, N \geq n$, and $Q_{c}$ such that

$$
W_{c} A_{c}=Q_{c} W_{c}, \quad \mu_{2}\left(Q_{c}\right)<0
$$

(ii) $A_{c}$ is Hurwitz stable if and only if there exists a full column rank matrix $W_{c} \in \mathcal{R}^{N \times n}, N \geq n$, and $Q_{c}$ such that

$$
W_{c} A_{c}=Q_{c} W_{c}, \quad \mu_{\infty}\left(Q_{c}\right)<0
$$

\footnotetext{
${ }^{1}$ The published paper in TAC omitted erroneously in this statement the assumption of distinct eigenvalues. All theorems and lemmas in the published paper contain the correct assumption.
}

(iii) $A_{d}$ is Schur stable if and only if there exists a full column rank matrix $W_{d} \in \mathcal{R}^{N \times n}, N \geq n$, and $Q_{d}$ such that

$$
W_{d} A_{d}=Q_{d} W_{d}, \quad\left\|Q_{d}\right\|_{2}<1
$$

(iv) $A_{d}$ is Schur stable if and only if there exists a full column rank matrix $W_{d} \in \mathcal{R}^{N \times n}, N \geq n$, and $Q_{d}$ such that

$$
W_{d} A_{d}=Q_{d} W_{d}, \quad\left\|Q_{d}\right\|_{\infty}<1
$$

Remark 1: Notice that $W_{c} A_{c}=Q_{c} W_{c}$ always imply that $W_{d}(h) A_{d}(h)=Q_{d}(h) W_{d}(h)$ with $W_{d}(h)=W_{c}$ and $Q_{d}(h)=Z\left(Q_{c} h\right)$. However it is not true in general that $\mu_{\infty}\left(Q_{c}\right)<0$ implies $\left\|Q_{d}(h)\right\|_{\infty}<1$, unless $h$ is small. A counterexample is given in [8].

Notice that Lemma 1-(i) and 1-(iii) enforce the existence of a quadratic Lyapunov function $\left\|W_{c} x_{c}\right\|^{2}=x_{c}^{\prime} P_{c} x_{c}$, where $P_{c}=W_{c}^{\prime} W_{c}$ and $\left\|W_{d} x\right\|^{2}=x_{d}^{\prime} P_{d} x_{d}$, where $P_{d}=W_{d}^{\prime} W_{d}$, respectively. Indeed, in the continuous-time case

$$
A_{c}^{\prime} P_{c}+P_{c} A_{c}=W_{c}^{\prime}\left(Q_{c}+Q_{c}^{\prime}\right) W_{c}<0
$$

whereas in the discrete-time case

$$
A_{d}^{\prime} P_{d} A_{d}-P_{d}=W_{d}^{\prime}\left(Q_{d}^{\prime} Q_{d}-I\right) W_{d}<0
$$

Moreover, matrices $W_{c}$ and $W_{d}$ can be always chosen as square $n \times n$ matrices, given by $W_{c}=P_{c}^{1 / 2}, W_{d}=P_{d}^{1 / 2}$. A notable result concerning quadratic Lyapunov functions is that they are invariant under Padé transformations. This means that, given a Hurwitz stable matrix $A_{c}$ and $P>0$ satisfying

$$
A_{c}^{\prime} P+P A_{c}<0
$$

then

$$
A_{d}(h)^{\prime} P A_{d}(h)<P, \quad \forall h>0
$$

This results follows directly by inspection for Padé transformation of order 1. For higher orders transformations the result relies on the existence of common quadratic Lyapunov functions for commutative matrices, and from properties of the complex 1'st order transformation; see [11], [12]. In our case the two matrices that commute are $A_{c}$ and $\bar{A}_{c}(h)=$ $\left(I+A_{d}(h)\right)\left(A_{d}(h)-I\right)^{-1}$, see [12]. In conclusion, denoting with $\mathcal{P}_{c}$ the set of $P>0$ satisfying $A_{c}^{\prime} P+P A_{c}<0$ and with $\mathcal{P}_{d}(h)$ the set of $P>0$ satisfying $A_{d}(h)^{\prime} P A_{d}(h)<P$, it follows

$$
\mathcal{P}_{c} \subset \mathcal{P}_{d}(h), \quad \forall h>0
$$

On the other hand the Lyapunov functions associated with Lemma 1-(ii) and 1-(iv) are of polyhedral type, i.e. $\left\|W_{c} x_{c}\right\|_{\infty}$, resp. $\left\|W_{d} x_{d}\right\|_{\infty}$, where the number of vertices of the polyhedra is $2 N$. In general $N>n$, and the minimal $N$ for which (6), (8) are verified depends on the location of the eigenvalues of $A_{c}, A_{d}$ in the complex plane. It can be proved, see [13], that for a matrix $A_{c}$ with distinct eigenvalues a necessary and sufficient condition for $N=n$ is that the complex eigenvalues $\lambda=-\alpha+j \beta, \alpha>0$, belong to the sector $|\beta| \alpha^{-1}<1$. 


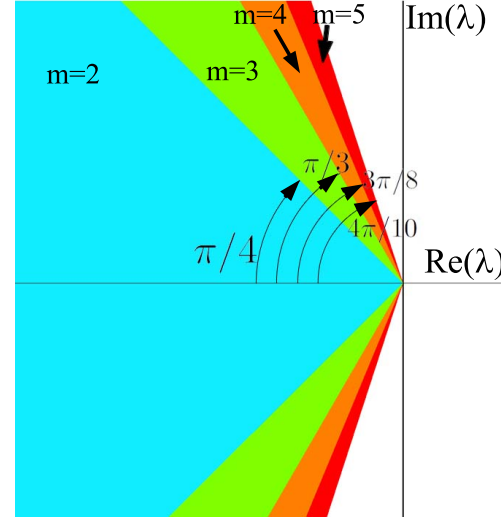

Figure 1. The sectors $\mathcal{S}_{c}(m)$ for $m=2$ (angle $\pi / 4$ ), $m=3$ (angle $\pi / 3$ ), $m=4$ (angle $3 \pi / 8$ ) and $m=5$ (angle $4 \pi / 10$ ).

\section{MAIN RESULT}

In this section we analyze the properties and relation of the sets $\mathcal{W}_{c}$, [resp. $\mathcal{W}_{d}(h)$ ] of matrices $W_{c}$, [resp. $W_{d}(h)$ ] satisfying (6), [resp. (8)]. First of all, we recall a pair of results available in the literature, [14], [15] for the continuous-time and discrete-time case, respectively.

Lemma 2: Consider a Hurwitz stable matrix $A_{c}$, with distinct eigenvalues, with $n_{r}$ real and $2 n_{c}$ complex eigenvalues. For each pair of conjugate complex eigenvalue $\lambda_{i}=\alpha_{i} \pm j \beta_{i}$, $i=1,2, \cdots, n_{c}$, take an integer $m_{i}$ such that $\lambda_{i}$ lies in the sector $\mathcal{S}_{c}\left(m_{i}\right)$, where

$$
\mathcal{S}_{c}(m)=\left\{\lambda=-\alpha+j \beta: \quad \alpha>0,|\beta|<\frac{\sin \left(\frac{\pi}{m}\right)}{1-\cos \left(\frac{\pi}{m}\right)} \alpha\right\} .
$$

Then there exists $W_{c} \in \mathcal{R}^{N \times n}$ and $Q_{c} \in \mathcal{R}^{N \times N}$, with $N=$ $\sum_{i=1}^{k} m_{i}+n_{r}$, satisfying (6).

In Figure 1, the sectors $\mathcal{S}_{c}(m)$ are drawn for $m=2$ (angle $\pi / 4$ ), $m=3$ (angle $\pi / 3$ ), $m=4$ (angle $3 \pi / 8$ ) and $m=5$ (angle $4 \pi / 10$ ).

Lemma 3: Consider a Schur stable matrix $A_{d}$, with distinct eigenvalues, with $n_{r}$ real and $2 n_{c}$ complex eigenvalues. For each pair of conjugate complex eigenvalue $\lambda_{i}=\sigma_{i} \pm j \omega_{i}$, $i=1,2, \cdots, n_{c}$, take an integer $m_{i}$ such that $\lambda_{i}$ lies in the interior of the regular polygon $\mathcal{P}_{\text {ol }}\left(m_{i}\right)$, where

$$
\mathcal{P}_{\text {ol }}(m)=\operatorname{int} \operatorname{conv}\left\{e^{j \frac{p \pi}{m}}\right\}_{p=0}^{2 m-1} .
$$

Then there exists $W_{d} \in \mathcal{R}^{N \times n}$ and $Q_{d} \in \mathcal{R}^{N \times N}$, with $N=$ $\sum_{i=1}^{k} m_{i}+n_{r}$, satisfying (8).

In Figure 2 the polygons $\mathcal{P}_{o l}(m)$ are depicted for $m=2$ (square), $m=3$ (hexagon), $m=4$ (octagon), $m=5$ (decagon). The two Lemmas above have been shown to be valid also in case of multiple eigenvalues. However, for the sake of simplicity, we assume that the eigenvalues are distinct, so facilitating the construction of $W_{c}$ and $W_{d}$. As shown in [14], matrix $W_{c}$ can be constructed as follows. Let $T_{c}$ be the state-space transformation that puts $A_{c}$ in its real Jordan form,

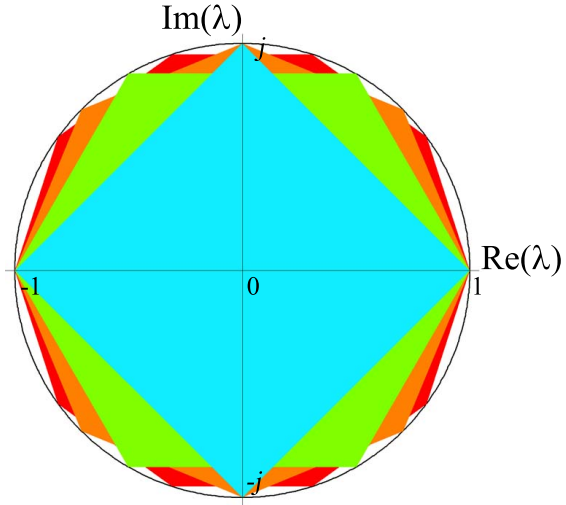

Figure 2. The polygons for $m=2$ (square), $m=3$ (hexagon), $m=4$ (octagon), $m=5$ (decagon).

i.e.

$$
T_{c} A_{c} T_{c}^{-1}=\left[\begin{array}{ccccc}
H_{c 1} & 0 & \cdots & 0 & 0 \\
0 & H_{c 2} & \cdots & 0 & 0 \\
\vdots & \vdots & \ddots & \vdots & \vdots \\
0 & 0 & 0 & H_{c n_{c}} & 0 \\
0 & 0 & 0 & 0 & R_{c}
\end{array}\right]
$$

where

$$
H_{c i}=\left[\begin{array}{cc}
-\alpha_{i} & \beta_{i} \\
-\beta_{i} & -\alpha_{i}
\end{array}\right]
$$

and $R_{c}$ is a $n_{r} \times n_{r}$ diagonal matrix accounting for the real eigenvalues. Moreover let, for $i=1,2, \cdots, n_{c}$ :

$$
\begin{aligned}
& W_{c i}=\left[\begin{array}{cc}
1 & 0 \\
\cos \left(\frac{\pi}{m_{i}}\right) & \sin \left(\frac{\pi}{m_{i}}\right) \\
\cos \left(\frac{2 \pi}{m_{i}}\right) & \sin \left(\frac{2 \pi}{m_{i}}\right) \\
\vdots & \vdots \\
\cos \left(\frac{\left(m_{i}-1\right) \pi}{m_{i}}\right) & \sin \left(\frac{\left(m_{i}-1\right) \pi}{m_{i}}\right)
\end{array}\right], \\
& Q_{c i}=\left[\begin{array}{ccccc}
x_{i} & y_{i} & 0 & 0 & 0 \\
0 & x_{i} & y_{i} & 0 & 0 \\
\vdots & \vdots & \ddots & \vdots & \vdots \\
0 & 0 & 0 & x_{i} & y_{i} \\
-y_{i} & 0 & 0 & 0 & x_{i}
\end{array}\right]
\end{aligned}
$$

where

$$
x_{i}=-\alpha_{i}-\frac{\beta_{i} \cos \left(\frac{\pi}{m_{i}}\right)}{\sin \left(\frac{\pi}{m_{i}}\right)}, \quad y_{i}=\frac{\beta_{i}}{\sin \left(\frac{\pi}{m_{i}}\right)}
$$

Then, it is easy to verify that (6) is satisfied with

$$
\begin{aligned}
W_{c}= & {\left[\begin{array}{ccccc}
W_{c 1} & 0 & \cdots & 0 & 0 \\
0 & W_{c 2} & \cdots & 0 & 0 \\
\vdots & \vdots & \ddots & \vdots & \vdots \\
0 & 0 & \cdots & W_{c n_{c}} & 0 \\
0 & 0 & \cdots & 0 & I
\end{array}\right] T_{c}, } \\
Q_{c}= & {\left[\begin{array}{ccccc}
Q_{c 1} & 0 & \cdots & 0 & 0 \\
0 & Q_{c 2} & \cdots & 0 & 0 \\
\vdots & \vdots & \ddots & \vdots & \vdots \\
0 & 0 & \cdots & Q_{c n_{c}} & 0 \\
0 & 0 & \cdots & 0 & R_{c}
\end{array}\right] }
\end{aligned}
$$


Notice indeed that $\mu_{\infty}\left(Q_{c}\right)<0$ is forced by the assumption on the position of the eigenvalues that is equivalent to $x_{i}+\left|y_{i}\right|<$ 0 .

The computation of the polyhedral Lyapunov function $\left\|W_{d} x_{d}\right\|_{\infty}$ for the discrete-time system (3) follows the same lines and can be found in [15]. Let $T_{d}$ the state-space transformation that puts $A_{d}$ in its real Jordan form, i.e.

$$
T_{d} A_{d} T_{d}^{-1}=\left[\begin{array}{ccccc}
H_{d 1} & 0 & \cdots & 0 & 0 \\
0 & H_{d 2} & \cdots & 0 & 0 \\
\vdots & \vdots & \ddots & \vdots & \vdots \\
0 & 0 & \cdots & H_{d n_{c}} & 0 \\
0 & 0 & \cdots & 0 & R_{d}
\end{array}\right]
$$

where

$$
H_{d i}=\left[\begin{array}{cc}
\sigma_{i} & \omega_{i} \\
-\omega_{i} & \sigma_{i}
\end{array}\right]
$$

and $R_{d}$ is a $n_{r} \times n_{r}$ diagonal matrix accounting for the real eigenvalues. It can be verified that (8) is met with by choosing $W_{d}=W_{c} T_{c}^{-1} T_{d}$ and $Q_{d}$ as in (13), with $R_{c}$ replaced by $R_{d}$ and $Q_{c i}$ replaced by

$$
Q_{d i}=\left[\begin{array}{ccccc}
z_{1, i} & z_{2, i} & \cdots & z_{m-1, i} & z_{m, i} \\
-z_{m, i} & z_{1, i} & \cdots & z_{m-2, i} & z_{m-1, i} \\
\vdots & \vdots & \ddots & \vdots & \vdots \\
-z_{3, i} & -z_{4, i} & \cdots & z_{1, i} & z_{2, i} \\
-z_{2, i} & -z_{3, i} & \cdots & -z_{m, i} & z_{1, i}
\end{array}\right]
$$

where $z_{j, i}$ are such that $\sum_{j=1}^{m}\left|z_{j, i}\right|<1$. Notice that $\left\|Q_{d}\right\|_{\infty}<$ 1 is forced by the assumption on the position of the eigenvalues in the regular polygon $\mathcal{P}_{o l}\left(m_{i}\right)$. We are now interested in the action of the Padé transformation over the sectors $\mathcal{S}_{c}(m)$ of the complex plane defined above. Our first result is a technical lemma, showing that, if $\lambda$ belongs to $\mathcal{S}_{c}(m)$ then its image under the Padé transformation belongs to $\mathcal{P}_{o l}(m)$.

Lemma 4: Let $m$ be a positive integer number, $\mathcal{S}_{c}(m)$ defined in (10) and $\mathcal{P}_{o l}(m)$ defined in (11). Fix a sampling time $h>0$ and consider $\mathcal{S}_{d}(m, h)$ the image of $\mathcal{S}_{c}(m)$ under the Padé transformation (2), i.e.

$$
\mathcal{S}_{d}(m, h)=\left\{z=Z(\lambda h) Z(-\lambda h)^{-1}, \lambda \in \mathcal{S}_{c}(m)\right\}
$$

Then

$$
\mathcal{S}_{d}(m, h) \subseteq \mathcal{P}_{o l}(m) .
$$

Proof. The Padé transformation can be written as

$$
z=Z(\lambda h) Z(-\lambda h)^{-1}=\prod_{i=1}^{n_{r}} \frac{e_{i 1}+\lambda}{e_{i 1}-\lambda} \prod_{i=1}^{n_{c}} \frac{d_{i 2}+d_{i 1} \lambda+\lambda^{2}}{d_{i 2}-d_{i 1} \lambda+\lambda^{2}}
$$

where $n_{r}$ is the number of real roots of $Z(\lambda h)$, and $2 n_{c}$ the number of complex conjugate roots of $Z(\lambda h)$, with $n_{r}+2 n_{c}=$ $p$ [12]. On the other hand, it is well known, see [16], that the polynomial $Z(\lambda h)$ is Hurwitz, so that all coefficients are positive, in particular $e_{i 1}>0$ and $d_{i 2}>0$. The proof is split into three parts.

(i) First we prove that, if $\lambda \in \mathcal{S}_{c}(m)$ then

$$
z_{i}(\lambda)=\frac{e_{i 1}+\lambda}{e_{i 1}-\lambda} \in \mathcal{P}_{o l}(m)
$$

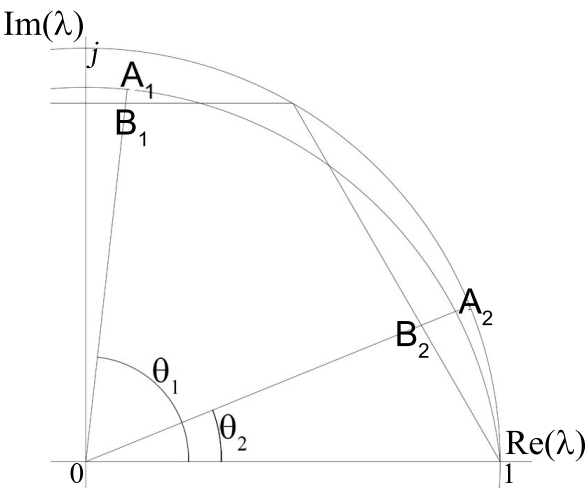

Figure 3. Proof: Existence of $A_{2}$.

We split $\mathcal{S}_{c}(m)$ in a family of lines

$$
\Lambda_{s}:=\{-\alpha+j \beta \mid \beta=s \alpha, \alpha>0\},
$$

indexed by $s \in(-r, r)$, where

$$
r=\frac{\sin \left(\frac{\pi}{m}\right)}{1-\cos \left(\frac{\pi}{m}\right)} .
$$

Our goal is to prove that $z_{i}\left(\Lambda_{s}\right) \subset \mathcal{P}_{o l}(m)$. Observe that $z_{i}$ is a Möbius transformation, thus it sends lines and circles into lines and circles. In our case, the image $z_{i}\left(\Lambda_{s}\right)$ is thus either an arc of circle or a segment. We compute $\lim _{\alpha \rightarrow 0} z_{i}(-\alpha+j s \alpha)=1$ and $\lim _{\alpha \rightarrow+\infty} z_{i}(-\alpha+j s \alpha)=-1$. Also the intersection with the imaginary axis can be computed, that is $j \psi(s)$ with

$$
\psi(s):=\frac{s}{1+\sqrt{1+s^{2}}} .
$$

Thus the image of $\Lambda_{s}$ via $z_{i}$ is an arc on the complex plane connecting 1 to $j \psi(s)$ to -1 (the extreme points 1 and -1 being excluded). Notice that $\psi(s)$ is an increasing function, thus the images of $\Lambda_{s}$ via $z_{i}$ are "ordered": given a fixed real component $x \in[-1,1]$, the corresponding imaginary component $y$ such that $x+j y \in z_{i}\left(\Lambda_{s}\right)$ is an increasing function with respect to $s$. For this reason, if $z_{i}\left(\Lambda_{s_{1}}\right) \subset$ $\mathcal{P}_{o l}(m)$ and $z_{i}\left(\Lambda_{s_{2}}\right) \subset \mathcal{P}_{\text {ol }}(m)$, then $z_{i}\left(\Lambda_{s}\right) \subset \mathcal{P}_{\text {ol }}(m)$ when $s_{1}<s<s_{2}$. We can thus restrict ourselves to prove that $z_{i}\left(\Lambda_{-r}\right) \subset \mathcal{P}_{o l}(m), z_{i}\left(\Lambda_{r}\right) \subset \mathcal{P}_{o l}(m)$. For symmetry with respect to the real axis, we can moreover restrict ourselves to prove that $z_{i}\left(\Lambda_{r}\right) \subset \mathcal{P}_{\text {ol }}(m)$. This is equivalent to prove that the arc connecting 1 to $j \psi(r)$ to -1 is contained in the regular polygon $\mathcal{P}_{o l}(m)$ (the extreme points 1 and -1 being excluded). Since we are dealing with a bilinear transformation, $z_{i}\left(\Lambda_{r}\right)$ is symmetric with respect to the imaginary axis. We can thus restrict ourselves to the part of the arc joining 1 to $j \psi(r)$ (the extreme point 1 being excluded). With this goal, we first observe that the arc is contained in the unit circle.

The slope of the arc in 1 can be computed deriving $z_{i}(-\alpha+j r \alpha)$ for $\alpha \rightarrow 0$. The slope is exactly $r_{C}=-r$, and it coincides with the slope of the first edge of the polygon $\mathcal{P}_{o l}(m)$, joining 1 to $e^{j \frac{\pi}{m}}$. Consider now the vector $z_{i}(-\alpha+j r \alpha)-1$ : its slope is always bigger than $-r$. Indeed,

$$
z_{i}(-\alpha+j r \alpha)-1=\frac{-2 \alpha\left(e_{i 1}+\alpha+r^{2} \alpha-j r e_{i 1}\right)}{\left(e_{i 1}+\alpha\right)^{2}+\alpha^{2} r^{2}}
$$


so that

$$
\frac{\operatorname{Im}\left(z_{i}(-\alpha+j r \alpha)-1\right)}{\operatorname{Re}\left(z_{i}(-\alpha+j r \alpha)-1\right)}=-\frac{r e_{i 1}}{e_{i 1}+\alpha\left(1+r^{2}\right)}>-r,
$$

recalling that $\alpha>0$ and $e_{i 1}>0$. We consider the equation of the arc in polar coordinate $\rho(\theta)$ with $\theta \in\left(0, \frac{\pi}{2}\right]$ and $\rho$ the corresponding radius. The function $\rho(\theta)$ is decreasing, due to geometrical observations Assume that there exists a point $A_{1}=\rho_{1} e^{j \theta_{1}}=z_{i}\left(-\alpha_{1}+j r \alpha_{1}\right) \notin \mathcal{P}_{o l}(m)$. If $\theta_{1} \notin\left(0, \frac{\pi}{m}\right]$, we prove that there exists another point $A_{2}=\rho_{2} e^{j \theta_{2}}=z_{i}\left(-\alpha_{2}+\right.$ $\left.j r \alpha_{2}\right) \notin \mathcal{P}_{o l}(m)$ such that $\theta_{2} \in\left(0, \frac{\pi}{m}\right]$. See Figure 3. Consider the unique point $B_{1}=\rho_{B} e^{j \theta_{1}}$ that is the intersection of the boundary of $\mathcal{P}_{o l}(m)$ with the segment joining 0 to $A_{1}$. We have $\rho_{B}<\rho_{1}$ because $A_{1}$ is outside $\mathcal{P}_{\text {ol }}(m)$. Compute now $k$ such that $\theta_{1} \in\left(\frac{k \pi}{m}, \frac{(k+1) \pi}{m}\right]$. Then the point $B_{2}=\rho_{B} e^{j\left(\theta_{2}\right)}$ with $\theta_{2}=\theta_{1}-\frac{k \pi}{m}$, lies on the boundary of $\mathcal{P}_{o l}(m)$ because of invariance of the boundary with respect to rotations of angle $\frac{k \pi}{m}$. Consider now the point $A_{2}=\rho_{2} e^{j \theta_{2}}$ with $\rho_{2}=\rho\left(\theta_{2}\right)$. It lies on the arc of circle and is outside $\mathcal{P}_{\text {ol }}(m)$, since $\rho_{2}$ is given by the image of $\rho($.$) . Moreover \rho_{2}>\rho_{1}$ because $\rho($. is decreasing and $\theta_{1}>\theta_{2}$. Recalling that $\rho_{2}>\rho_{1}>\rho_{B}$, we have that $A_{2}$ is outside the polygon. We now have a point $A_{2}$ belonging to the arc with $\theta_{2} \in\left(0, \frac{\pi}{m}\right]$ that is outside the polygon $\mathcal{P}_{\text {ol }}(m)$, but inside the circle. Then the vector $A_{2}-1$ to 1 has a slope that is strictly more negative than $-r$. But $A_{2} \in z_{i}\left(\Lambda_{r}\right)$, thus $A_{2}-1=z_{i}(-\alpha+j r \alpha)-1$ for some $\alpha$, and we have proved that this vector's slope is bigger than or equal to $-r$. This is a contradiction.

(ii) Now we consider the function

$$
z_{i}(\lambda)=\frac{d_{i 2}+d_{i 1} \lambda+\lambda^{2}}{d_{i 2}-d_{i 1} \lambda+\lambda^{2}}
$$

We assume $\lambda \in \Lambda_{s} \subset \mathcal{S}_{c}(m)$, that is $\lambda=-\alpha+j s \alpha$ with $|s|<r$ where $r$ is given by (15). We define

$$
\bar{\lambda}(\lambda)=\frac{z_{i}(\lambda)-1}{z_{i}(\lambda)+1}
$$

Notice that

$$
z_{i}(\lambda)=\frac{1+\bar{\lambda}(\lambda)}{1-\bar{\lambda}(\lambda)}
$$

After some manipulations it follows

$$
\frac{\operatorname{Im}(\bar{\lambda}(\lambda))}{\operatorname{Re}(\bar{\lambda}(\lambda))}=-s\left(\frac{d_{i 2}-\alpha^{2}\left(1+s^{2}\right)}{d_{i 2}+\alpha^{2}\left(1+s^{2}\right)}\right) .
$$

Recalling that $d_{i 2}>0$, we have

$$
\left|\frac{\operatorname{Im}(\bar{\lambda}(\lambda))}{\operatorname{Re}(\bar{\lambda}(\lambda))}\right| \leq|s|,
$$

thus $\bar{\lambda}(\lambda) \in \Lambda_{\bar{s}} \subset \mathcal{S}_{c}(m)$ for a certain $\bar{s}$ satisfying $|\bar{s}| \leq$ $|s|$. We can now apply the proof in Part (i) to $\bar{\lambda} \in \mathcal{S}_{c}(m)$, observing that the expression of $z_{i}$ with respect to $\bar{\lambda}$ is of kind (14). Thus $z_{i}(\lambda) \in \mathcal{P}_{\text {ol }}(m)$.

(iii) Finally notice that

$$
z=Z(\lambda h) Z(-\lambda h)^{-1}=\prod_{i=1}^{n_{r}+2 n_{c}} z_{i}
$$

where $z_{i} \in \mathcal{P}_{\text {ol }}(m)$. We just miss to show that $z \in \mathcal{P}_{\text {ol }}(m)$ as well. We prove this by showing that the set $\mathcal{P}_{\text {ol }}(m)$ is

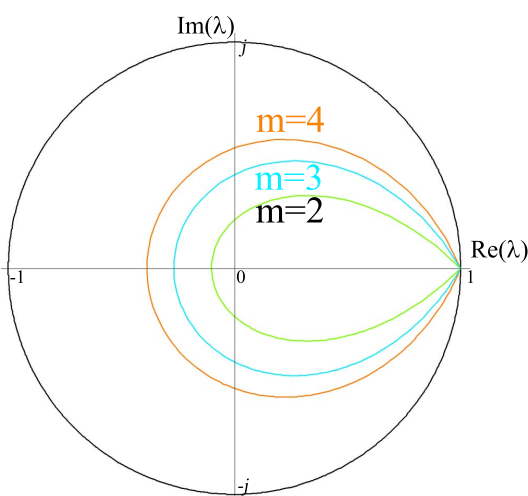

Figure 4. The vector $z=Z(\lambda h) Z(-\lambda h)^{-1}$ (with $p=2$ ) as a function of $\alpha>0$, for $\lambda=-\alpha(1+j r), h=1$ and $m=2,3,4$.

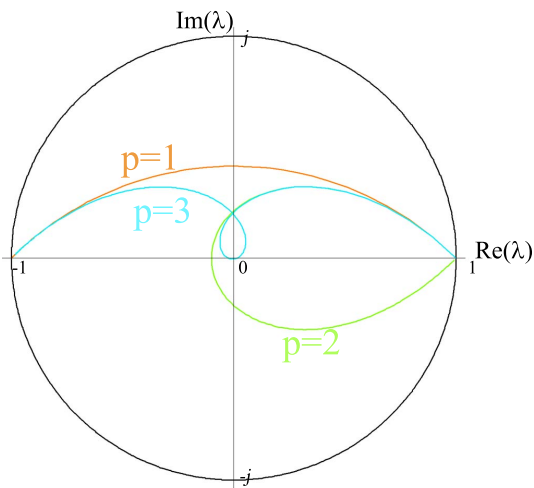

Figure 5. The vector $z=Z(\lambda h) Z(-\lambda h)^{-1}$ as a function of $\alpha>0$, for $\lambda=-\alpha(1+j r), h=1$ and Padé of order $p=1,2,3$.

closed under multiplication. Indeed, take $\xi_{1} \in \mathcal{P}_{o l}(m)$ and $\xi_{2} \in \mathcal{P}_{\text {ol }}(m)$. Each of them can be written as a convex combination of two vertices of the polygon, i.e.

$$
\xi_{1}=a_{1} e^{j \theta_{11}}+a_{2} e^{j \theta_{12}}, \quad \xi_{2}=b_{1} e^{j \theta_{21}}+b_{2} e^{j \theta_{22}}
$$

with $a_{i}, b_{i} \geq 0, a_{1}+a_{2}<1, b_{1}+b_{2}<1$ and $\theta_{i j}=\frac{k_{i j} \pi}{m}$ for $k_{i j} \in \mathbb{Z}$. Hence,

$$
\begin{aligned}
\xi_{1} \xi_{2}= & a_{1} b_{1} e^{j\left(\theta_{11}+\theta_{21}\right)}+a_{1} b_{2} e^{j\left(\theta_{11}+\theta_{22}\right)}+ \\
& +a_{2} b_{1} e^{j\left(\theta_{12}+\theta_{21}\right)}+a_{2} b_{2} e^{j\left(\theta_{12}+\theta_{22}\right)}
\end{aligned}
$$

with $a_{1} b_{1}+a_{1} b_{2}+a_{2} b_{1}+a_{2} b_{2}=\left(a_{1}+a_{2}\right)\left(b_{1}+b_{2}\right)<1$. In conclusion, $\xi_{1} \xi_{2} \in \mathcal{P}_{\text {ol }}(m)$.

In Figure 4 the 2 nd order Padé mapping $z=$ $Z(\lambda h) Z(-\lambda h)^{-1}$ is considered as a function of $\alpha>0$, when $h=1$ and $\lambda=-\alpha(1+j r)$, where $r$ is defined in (15), for $m=2,3,4$. Finally, in Figure 5, the mapping $z=Z(\lambda h) Z(-\lambda h)^{-1}$ is considered as a function of $\alpha>0$, when $h=1$ and $\lambda=-\alpha+j \alpha$ (i.e. $m=2$ ), taking the Padé transformation of order $p=1, p=2$ and $p=3$.

We are now in the position to prove the main result of the paper.

Theorem 1: Consider a Hurwitz stable matrix $A_{c}$ of dimension $n$ and its Padé discretization $A_{d}(h)$ of order $p$. Assume that all eigenvalues of $A_{c}$ are distinct. Let $n_{r}$ be the number of real negative eigenvalues, and $2 n_{c}$ be the number of pairs of complex eigenvalues $-\alpha_{i} \pm j \beta_{i}, i=1,2, \cdots, n_{c}$. For each 
pair of complex eigenvalues, let $m_{i}$ be an integer greater than one such that $-\alpha_{i} \pm j \beta_{i}$ belongs to the sector

$$
\left|\beta_{i}\right|<\frac{\sin \left(\frac{\pi}{m_{i}}\right)}{1-\cos \left(\frac{\pi}{m_{i}}\right)} \alpha_{i}
$$

Then there exist $W=W_{c}=W_{d}(h) \in \mathcal{R}^{N \times n}$, with $N=$ $\sum_{i=1}^{k} m_{i}+n_{r}, Q_{c} \in \mathcal{R}^{N \times N}, Q_{d}(h) \in \mathcal{R}^{N \times n}$, such that (6), (8) are met with, for all $h>0$.

Proof. First recall that the Padé transformation preserves the Jordan form of $A_{c}$ and $A_{d}$. Now take $T$ such that both $J_{c}=T A_{c} T^{-1}$ and $T A_{d}(h) T^{-1}$ are in the real Jordan form. Compute $W_{c}$ as in (12). For each pair of complex eigenvalue $\lambda_{i}=-\alpha_{i} \pm \beta_{i}$, the expression of $W_{c i}$ is uniquely by $m_{i}$ such that $-\alpha_{i} \pm \beta_{i} \in \mathcal{S}_{c}\left(m_{i}\right)$. Now consider the expression of $W_{d}(h)$, that is identical to (12), replacing each $W_{c i}$ with $W_{d i}$, accounting for the pair of complex eigenvalues $\mu_{i}=$ $\sigma_{i} \pm j \omega_{i}=Z\left(\lambda_{i} h\right) Z\left(-\lambda_{i} h\right)^{-1}$. Applying Lemma 4, we have that $\mu_{i}$ lies in the interior of the regular polygon $\mathcal{P}_{o l}\left(m_{i}\right)$, with the same $m_{i}$ of the eigenvalue $\lambda_{i}$ of the continuous system. As a consequence, the expression of $W_{d i}$ can be chosen to be identical to $W_{c i}$. Thus $W_{d}(h)=W_{c} T_{c}^{-1} T_{d}$. Since $T_{c}=T_{d}$, we have the conclusion.

Comment : The result above says that there always exists a common polyhedral Lyapunov function $\|W x\|_{\infty}$ for $A_{c}$ and $A_{d}(h)$. Moreover, the construction of $W$ is based on the matrix $A_{c}$ only, and the previous theorem shows $\|W x\|_{\infty}$ is a polyhedral Lyapunov function for $A_{d}(h)$ computed with a Padé approximation of any order $p$. As a consequence, the polyhedral Lyapunov function is common to $A_{c}$ and all Padé approximants, of any order $p$ and with any sampling time $h$. We recall that, however, there is a great difference between quadratic and polyhedral Lyapounov functions, as stated in [8]: indeed, all quadratic Lyapunov functions are preserved under any Padé discretization, while this is not more valid for polyhedral functions. The following counterexample is given in [8]. Consider the Hurwitz matrix

$$
A_{c}=\left[\begin{array}{cc}
-1 & 0 \\
-2.4 & -3
\end{array}\right]
$$

Hence, since $\mu_{\infty}\left(A_{c}\right)<0$ it is very simple to compute $W_{c}$ and $Q_{c}$ satisfying (6). Indeed, such equations are satisfied by $W_{c}=I$ and $Q_{c}=A_{c}$. Now, take $A_{d}(1)$ given by the $1^{\text {st }}$ order Padé approximation with $h=2$, namely

$$
A_{d}(1)=\left(I+A_{c}\right)\left(I-A_{c}\right)^{-1}=\left[\begin{array}{cc}
0 & 0 \\
-0.6 & -0.5
\end{array}\right]
$$

and notice that $Q_{d}=\left(I+Q_{c}\right)\left(I-Q_{c}\right)^{-1}$ satisfies $A_{d} W_{d}=$ $W_{d} Q_{d}$, with $W_{d}=W_{c}$. However, $\left\|Q_{d}\right\|_{\infty}>1$. From this the authors in [8] concluded that $W_{c}=I$ is not preserved. Nevertheless, using our result, it is possible to find another $W_{c}$ that is preserved. A choice is given by

$$
\begin{gathered}
W=\left[\begin{array}{cc}
-1 & 0 \\
1.2 & 1
\end{array}\right], Q_{c}=\left[\begin{array}{cc}
-1 & 0 \\
0 & -3
\end{array}\right], Q_{d}=\left[\begin{array}{cc}
0 & 0 \\
0 & -0.5
\end{array}\right] \\
\text { V. ConcLusions }
\end{gathered}
$$

In this paper it was shown that a continuous-time stable system and its associated sampled system via Padé transformation (of any order) always share a particular polyhedral
Lyapunov function. The consequence of our result is most pronounced for the discretisation of switched systems. It is in this context that our result may be important. It is well known that polyhedral Lyapunov function are non conservative in the analysis of stability of switched linear systems under arbitrary switching. Consequently, we believe that the result on polyhedral Lyapunov functions also shades some light on a possible approach to discretise switched linear systems; namely, if certain types of Lyapunov functions are common to all modes of the system, then stability of preserved under Padé discretisations. Despite this fact, much work remains to be done, and this work can take several possible directions. First, we can look for discretisation methods that preserve all polyhedral Lyapunov functions for sets of matrices irrespective of sampling rate and of order. This is difficult, but some progress along these lines has been made in the context of positive systems [2]. Another direction is to search for conditions on the the sampling time for which such Padé approximation preserves Lyapunov functions of a certain type.

\section{ACKNOWLEDGMENTS}

This paper has been partially supported by the Italian National Research Council (CNR) and by Science Foundation Ireland under grant number PI Award 07/IN.1/1901.

\section{REFERENCES}

[1] R. Shorten, S. Kumar, M. Corless, S. Solmaz, "On Padé Approximations and the Preservation of Quadratic Stability for Switched Linear Systems", Accepted for publication in Systems and Control Letters, 2011.

[2] A. Zappavigna, P. Colaneri, S. Kirkland, R. Shorten, "Essentially Negative News about Non-negative Matrices, Linear Algebra and its Applications", Submitted to Linear Algebra and its Applications, 2010.

[3] Ricardo G. Sanfelice, Andrew R. Teel, "Dynamical properties of hybrid systems simulators", Automatica, Vol. 46 , Issue 2, pp. 239-248, 2010.

[4] A. Pietrus, V. Veliov, "On the discretization of switched linear systems", Systems and Control Letters, Vol. 58, No. 6, pp. 395-399, 2009.

[5] F. Blanchini and S. Miani, Set theoretic methods in control, Birkhauser, Boston, 2008.

[6] G. Birkhoff, R.S. Varga, "Discretization errors for well-set Cauchy problems", Journal of Mathematics Physics, V. 44 N. 1, pp. 28-51, 1965.

[7] R.C. Ward, "Numerical computation of the matrix exponential with accuracy estimate", SIAM Journal on Numerical Analysis, Vol. 14, N. 4, pp. 600-610, 1977.

[8] T. Mori, T.V. Nguyen, Y. Mori, H. Kokame, "Preservation of Lyapunov functions under bilinear mapping", Automatica, Vol. 42, pp. 1055-1058, 2006.

[9] P. Falcone and F. Borrelli and J. Asgari and H. E. Tseng and D. Hrovat, "MPC-Based Yaw and Lateral Stabilization Via Active Front Steering and Braking Vehicle System Dynamics", Vehicle System Dynamics, Vol. 46, pp. 611-628, 2008.

[10] H. Kiendl, J. Adamy, P. Stelzner, "Vector norms as Lyapunov functions for linear systems", IEEE Transaction on Automatic Control, Vol. 439, pp. 839-842, 1995.

[11] K. S. Narendra, J. Balakrishnan, "A common Lyapunov function for stable LTI systems with commutative $A$ - matrices", IEEE Transaction on Automatic Control, Vol. 39, N. 712, 1994.

[12] S. Sajja, M. Corless, S. Solmatz, R. Shorten, "Preservation of common quadratic Lyapunov functions and Pade' approximation", IEEE Conference on Decision and Control, Atlanta, 2010.

[13] E.B. Castelan, J.C. Hennet, "On invariant polyhedra of continuous-time linear systems", IEEE Transactions on Automatic Control”, Vol. 38, N. 11, pp. 1680-1685, 1993.

[14] A. Polanski, "On infinity norms as Lyapunov functions for linear systems", IEEE Transaction on Automatic Control, Vol. 40, N. 7, pp. 1270-1274, 1995.

[15] F.K. Christophersen, M. Morari, "Further results on 'Infinity norms as Lyapunov functions for linear systems", IEEE Transaction on Automatic Control, Vol. 52, N. 3, pp. 547-553, 2007. 
[16] G.A. Baker Jr, P. Graves-Morris, "Padé Approximants", Vol. 59, Encyclopedia of Mathematics and Its Applications, Cambridge University Press, second edition, 1996. 